\title{
Improvement of left ventricular function in patients with persistent atrial tachyarrhythmia induced cardiomyopathy undergoing radiofrequency ablation
}

\author{
Xiangmin Shi, Zhaoliang Shan, Hongyang Guo, Yutang Wang
}

Department of Cardiology, PLA General Hospital, Beijing, China

Email: shixm301cardiac@hotmail.com

Received 25 September 2013; revised 29 October 2013; accepted 15 November 2013

Copyright (C) 2013 Xiangmin Shi et al. This is an open access article distributed under the Creative Commons Attribution License, which permits unrestricted use, distribution, and reproduction in any medium, provided the original work is properly cited. In accordance of the Creative Commons Attribution License all Copyrights (C) 2013 are reserved for SCIRP and the owner of the intellectual property Xiangmin Shi et al. All Copyright (C) 2013 are guarded by law and by SCIRP as a guardian.

\section{ABSTRACT}

Purpose: To investigate the alteration of left ventricular function in subjects with persistent atrial tachyarrhythmia induced cardiomyopathy (TIC) undergoing radiofrequency ablation, and to study the pathogenesis and effective treatment of TIC. Methods: A total of 25 cases with persistent atrial tachyarrhythmia and impaired left ventricular systolic function were studied (16 men and 9 women, aged $53.3 \pm 15.2$ years), and all subjects underwent electrophysiological study and radiofrequency ablation of atrial tachyarrhythmia under the guidance of CARTO system during 2006.9-2011.8. Indexes related to cardiac function, including left ventricular end diastolic diameter (LVEDD), left ventricular ejection fraction (LVEF), New York Heart Association functional classification (NYHA class), 6 minutes walking test (6MWT), Nterminal pro-brain natriuretic peptide (BNP) and 24 hours average heart rate (AHR), were analyzed at the time point of 7 days, 3 and 6 months after the procedure as well as 1 day before ablation. Results: No refractory atrial arrhythmia recurred in all cases after ablation, compared with LVEDD (51.7 $\pm 4.5 \mathrm{~mm})$, LVEF $(39.0 \% \pm 4.3 \%)$, number of patients with NYHA class IV and III $(\mathrm{n}=17), 6 \mathrm{MWT}(212 \pm 56 \mathrm{~m})$, BNP (3622 $\pm 1860 \mathrm{ng} / \mathrm{L})$ and AHR (112.5 $\pm 23.2 \mathrm{bpm})$ before ablation, the index of LVEDD $(45.2 \pm 3.3 \mathrm{~mm}$; $41.7 \pm 2.5 \mathrm{~mm}$; $40.5 \pm 3.1 \mathrm{~m})$, BNP (2429 $\pm 1355 \mathrm{ng} / \mathrm{L}$; $1530 \pm 866 \mathrm{ng} / \mathrm{L} ; 1300 \pm 520 \mathrm{ng} / \mathrm{L})$, total number of patients of NYHA class IV and III $(n=11 ; 3 ; 2)$ and AHR (73.3 \pm 15.3 bpm; $68.7 \pm 13.5$ bpm; $66.3 \pm 13.6$ bpm) significantly decreased $(P<0.05)$, LVEF (45.6 \pm $3.5 \% ; 51.5 \pm 2.7 \% ; 53.5 \pm 3.1 \%)$ and 6MWT (262 \pm $47 \mathrm{~m} ; 305 \pm 37 \mathrm{~m} ; 313 \pm 41 \mathrm{~m})$ greatly increased $(P<$ 0.05 ) in 7 days, 3 and 6 months after ablation respectively. There was a statistical difference between 7 days and 3 or 6 months after ablation in above-mentioned indexes $(P<0.05)$ except AHR $(P>0.05)$, no significant difference existed between 3 and 6 months in all indexes $(P>0.05)$. Conclusion: long-lasting atrial arrhythmia with rapid ventricular response could impair left ventricle function, which could be reversed within weeks after successful ablation and restoration of sinus rhythm.

Keywords: Tachyarrhythmia-Induced Cardiomyopathy; Radiofrequency Ablation; Left Ventricular Dysfunction; Sinus Rhythm

\section{INTRODUCTION}

Atrial tachyarrhythmia is one of the most common encountered problems in clinical practice, long lasting of which could result in tachycardia-induced cardiomyopathy (TIC) [1], presenting cardiac enlargement and impaired left ventricular systolic function, similar to that of idiopathic dilated cardiomyopathy (IDC) [2]. Meanwhile, patients with IDC are susceptible to develop atrial arrhythmia due to increased intra-atrium pressure [3], which in turn further deteriorate the cardiac function and cause refractory heart failure [4]. The aim of this study was to investigate the impact of atrial tachyarrhythmia on left ventricular function and its alteration after elimination of atrial arrhythmia by means of radiofrequency ablation in 25 patients who already received optimized medication for heart failure, and to study the role of ablation in the treatment of TIC.

\section{METHODS}

\subsection{Study Population}

This study included 25 patients with persistent atrial ar- 
rhythmia with rapid ventricular response (16 men and 9 women, aged, $53.3 \pm 15.2$ years) referred for radiofrequency ablation during 2006.9-2011.8, number of subjects with atrial fibrillation (AF), atrial flutter and atrial tachycardia were 15, 7 and 3 respectively. All patients had documented atrial tachyarrhythmia refractory to at least 2 kinds of anti-arrhythmic drugs, of whom 4 subjects suffered IDC prior to the development of arrhythmia, the rest 21 cases were not detected underlying structural heart disease at the first clinical visit and gradually manifested symptoms of heart failure with the prolongation of arrhythmia. Patients with hyperthyroidism, old myocardial infarction, rheumatic valve disease, chronic obstructive pulmonary disease (COPD) were not recruited in this study. Most of patients were treated with optimal medication of heart failure, including diuretics, digoxin, $\beta$ receptor antagonist, angiotension convert enzyme inhibitor (ACEI) or angiotension II receptor antagonist (ARB) and vasodilators. Informed consent was obtained from all subjects prior to procedure. Baseline characteristics of patients undergoing radiofrequency ablation are shown in Table $\mathbf{1}$.

\subsection{Electrophysiological Study of Atrial Fibrillation}

Patients with persistent AF were kept on oral anticoagulation with warfarin 1 month prior to procedure, warfarin was withdrawn 3 days before ablation, all anti-arrhythmic drugs were discontinued at least five half-lives. Every patient after giving informed consent underwent an electrophysiological study in a fasting and conscioussedated state. One decapolar mapping catheter (Biosense

Table 1. Baseline characteristics of patients undergoing radiofrequency ablation.

\begin{tabular}{cc}
\hline Characteristics & Values \\
\hline Age (years) & $53.3 \pm 15.2$ \\
Men (\%) & $16(64 \%)$ \\
LVEDD (mm) & $51.7 \pm 4.5$ \\
LVEF (\%) & $39.0 \pm 4.3$ \\
Average heart rate (bpm) & $112.5 \pm 23.2$ \\
Duration of arrhythmia (years) & $2.8 \pm 1.4$ \\
Comorbidities & $8(32 \%)$ \\
Hypertension (n) & $5(20 \%)$ \\
(n) & $5(20 \%)$ \\
OSAHS (n) & $6(24 \%)$ \\
Diabetes mellitus (n) & $4(16 \%)$ \\
Idiopathic dilated cardiomyopathy (n) & \\
Treatment & $21(84 \%)$ \\
Diuretics (n) & $20(80 \%)$ \\
Inotropes (n) & $23(92 \%)$ \\
Beta blockers (n) & $23(92 \%)$ \\
ACEI or ARB (n) & $25(100 \%)$ \\
Vasodilators (n) &
\end{tabular}

Webster, Diamond Bar, CA) was positioned in the coronary sinus (CS) through the right jugular vein access, using the standard Brockenbrough technique [5], atrial transseptal puncture was performed under fluoroscopic guidance and two $\mathrm{L}_{1}$-type Swartz sheathes (St Jude Medical, Minneapolis, MN) were transseptally introduced into LA via right femoral vein. Intravenous heparin $5000 \mathrm{U}$ was administered immediately after atrial transseptal puncture and followed $1000 \mathrm{U} / \mathrm{h}$ to maintain an activated clotting time (ACT) of $300-350 \mathrm{~s}$, the ACT level was monitored every $30 \mathrm{~min}$. In addition, heaprinized saline solution was continuously infused through the transseptal sheath $(3 \mathrm{~mL} / \mathrm{min})$ to avoid formation of thrombi or air emboli. Selective PV venography was performed to identify all PV ostia. One decapolar circular mapping catheter (Lasso, Biosense Webster) was placed at the ostium of each PV to record PV potentials.

\subsection{Circumferential Pulmonary Vein Isolation (Cpvi)}

The procedure of CPVI was performed under the guidance of CARTO system (Biosense Webster), a $3.5 \mathrm{~mm}$ saline-irrigated catheter (Navi-star, Thermocool, Biosense Webster) was transseptally advanced into LA via Swartz sheathe, LA geometry was reconstructed and each PV ostium was tagged on it. Pulmonary vein isolation was performed 5 to $10 \mathrm{~mm}$ outside of the PV ostia. Ipsilateral pair of left and right PVs were isolated in one circumferential lesion. Radiofrequency (RF) current was delivered point by point at a target temperature $43^{\circ} \mathrm{C}$, maximum power of $35 \mathrm{~W}$, and an infusion rate of 17 $\mathrm{mL} / \mathrm{min}$. If AF could not terminate after the procedure of CPVI, direct current cardioversion was performed to restore sinus rhythm. The end point of CPVI was electrical isolation of PV potentials, which was confirmed by Lasso catheter mapping during sinus rhythm or isoproterenol infusion after $30 \mathrm{~min}$. The end point was bidirectional conduction block between LA and PVs. If AF was not terminated by above-mentioned ablation, additional ablation lines were created, including a bottom line connecting both inferior PVs and/or a mitral isthmus line. Linear ablation at cavotricuspid isthmus was created in patients with documented or inducible cavotricuspid isthmus dependent atrial flutter, until bidirectional isthmus block was achieved.

\subsection{Electrophysiological Study and Ablation of Atrial Flutter and Atrial Tachycardia}

For patients with atrial flutter, two quadripolar catheters (2-5-2 mm, DAIG, St Jude Medical Inc, St Paul, MN, USA) were positioned at the his bundle and right atrium via the left femoral vein access, One decapolar mapping catheter (Biosense Webster, Diamond Bar, CA) was po- 
sitioned in the coronary sinus (CS) through the right jugular vein access. Activation sequence of atrial flutter entrapment mapping was assessed by a 20-pole electrode halo catheter (Biosense Webster, Diamond Bar, CA). The electrophysiological definition of isthmus dependent atrial flutter was demonstrated by the standard criteria for entrainment mapping, a deflectable $5 \mathrm{~mm}$ tipped quadripolar catheter was used as the ablation catheter. RF ablation started at the ventricular side of inferolateral tricuspid annulus (TA) and end point was bidirectional block of isthmus conduction. Energy was delivered at 50 $\mathrm{W}$ targeting $60^{\circ} \mathrm{C}$, this was followed by a series of discrete applications along a line between the TA and IVC orifice. A bidirectional isthmus block was demonstrated by pacing technique which was performed at coronary ostium and the low lateral right atrium. The line of block was reevaluated $30 \mathrm{~min}$ after the catheter ablation. For patients with atrial tachycardia (AT), we used CARTO system to obtain three-dimensional mapping during AT and ablated at the earliest activation sites or within superior vena cava (SVC) (Figure 1), a temperature-controlled, 4-mm-tip ablating catheter (maximal temperature, $60^{\circ} \mathrm{C}$; maximal power, $30 \mathrm{~W}$ ) with titration of power and temperature was used for ablation. When a left-sided origin was suspected, access to the left atrium for mapping and ablation was obtained transseptally using a Brockenbrough needle. Acute procedural success was defined by the absence of tachycardia or dissociation of SVC potentials with right atrial activities in arrhythmia with SVC origin (Figure 2) 30 min after ablation despite infusion of isoproterenol and burst atrial pacing.

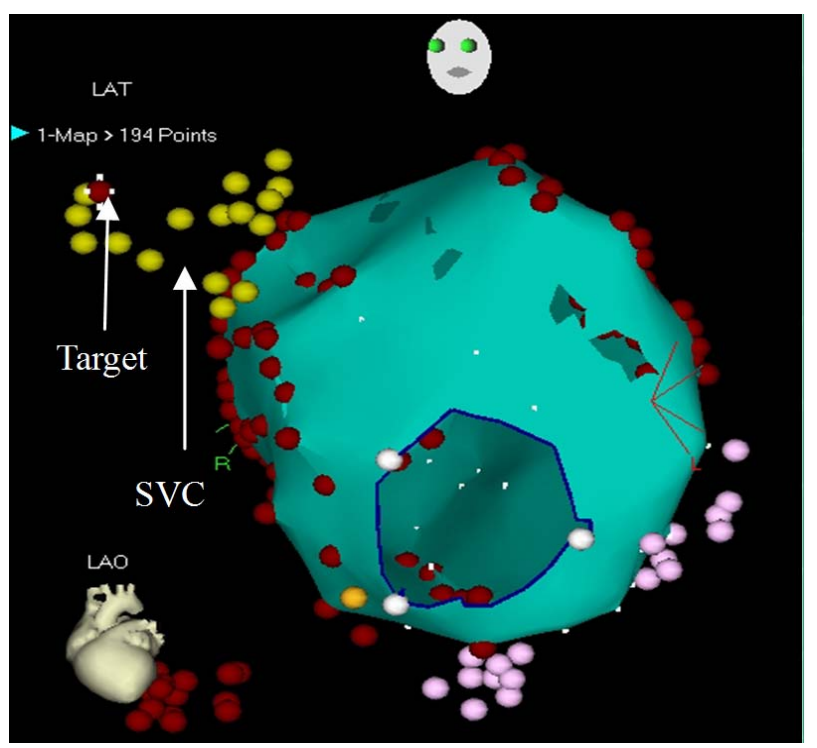

Figure 1. Successful ablation site in CARTO mapping system (LAO projection), atrial tachycardia was converted into sinus rhythm just after the ablation of target site located in the free wall of SVC. LAO: left anterior oblique SVC: superior vena cava.

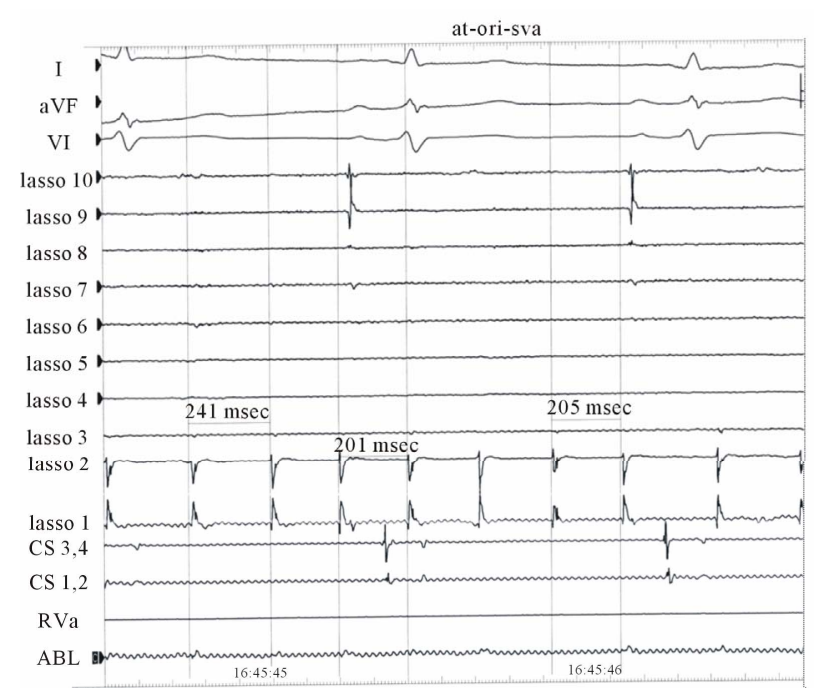

Figure 2. ECG and intracardiac mapping after successful ablation: SR was restored in surface ECG after ablation, irregular rapid firing (arrow indicated) was recorded by the distal circular catheter in SVC, meanwhile, SR recorded by the proximal circular catheter in HRA, indicating SVC-HRA exit block. (SR: sinus rhythm; Lasso: circular catheter; CS: coronary sinus; RVa: right ventricular apex; HRA: high right atrium; ABL: ablation catheter).

\subsection{Postoperative Care and Follow-Up}

After the procedure, oral anticoagulation with warfarin was resumed and the normalized ratio (INRs) was maintained in the therapeutic range for subjects with $\mathrm{AF}$, meanwhile, oral aspirin $(75-100 \mathrm{mg} /$ day) recommended for 1 month to prevent thromboembolic complications for patients with atrial flutter and atrial tachycardia. Follow up was at 7 days, 3 and 6 months, all medication for heart failure continued during the period of follow up. Indexes related to cardiac function, including left ventricular end diastolic diameter (LVEDD), left ventricular ejection fraction (LVEF), New York Heart Association functional classification(NYHA class), 6 minutes walking test (6MWT), and N-terminal pro-brain natriuretic peptide (BNP), were obtained 1 day before ablation and at 7 days, 3 and 6 months after the procedure.

\subsection{Statistical Analysis}

Continuous variables were expressed as mean $\pm \mathrm{SD}$ and categorical variables as proportions (\%). Data were analyzed with unpaired $t$-test, categorical variables were compared with $\chi^{2}$ tests. A value of $<0.05$ was considered statistically significant. All analyses were performed using SPSS 12.0.

\section{RESULTS}

\subsection{Acute Procedure Result}

CPVI was performed in all 15 AF subjects, of which 5 
AF converted into sinus rhythm spontaneously during the process of CPVI and the other 10 cases required direct current cardioversion to restore SR, bottom line and cavotricuspid line were created in 4 and 3 cases respectively. 7 atrial flutter patients underwent right atrial cavotricuspid isthmus linear ablation, of which 6 were counter clockwise $(\mathrm{CCW})$ and 1 was clockwise $(\mathrm{CW})$ isthmus-dependent activation. For patients with atrial tachycardia, 2 cases originated from crista terminalis (CT) and the other one arising from superior vena cava (SVC). SVC isolation (SVCI) was achieved under the guidance of CARTO system (Figure 1) and Persistent rapid foci firing within SVC still remained in the presence of sinus rhythm restored after SVCI (Figure 2). No major complication occurred except mild pericardial effusion in 2 patients receiving CPVI and transient phrenic nerve palsies in 1 AT case undergoing ablation within SVC (Table 2). In the follow up, $3 \mathrm{AF}$ and $1 \mathrm{AT}$ subjects presented recurrence of paroxysmal atrial tachyarrhythmia within 1 month after procedure, which were controlled by intravenous administration of amiodarone.

\subsection{Alteration of Heart Function in the Follow-Up}

All subjects with tachycardia induced cardiomyopathy (TIC) continued unchanged medication during the 6 months follow-up, compared with indexes of heart function one day before procedure, LVEDD, NYHA class, BNP, 6MWT and LVEF significantly improved one week after ablation, and demonstrated further improvement in the follow-up, although there was no statistical difference between 3 and 6 months (Table 3), the trend of improvement could be found.

\section{DISCUSSION}

Tachycardia-induced cardiomyopathy (TIC) is deteriorating in ventricular function secondary to sustained tachycardia, which can lead to dilated cardiomyopathy and

Table 2. Comparison of different atrial tachyarrhythmia ablation.

\begin{tabular}{cccc}
\hline & $\begin{array}{c}\text { Procedure } \\
\text { time }(\min )\end{array}$ & $\begin{array}{c}\text { Fluoroscopic time } \\
(\mathrm{min})\end{array}$ & $\begin{array}{c}\text { Complication } \\
(\mathrm{n})\end{array}$ \\
\hline $\mathbf{A F}(\mathrm{n}=15)$ & $156 \pm 43$ & $63 \pm 17$ & Pericardial effusion(2) \\
Af $(\mathrm{n}=7)$ & & & 0 \\
$\mathrm{CCW}(\mathrm{n}=6)$ & $52 \pm 18$ & $28 \pm 11$ & 0 \\
$\mathrm{CW}(\mathrm{n}=1)$ & 48 & 25 & 0 \\
AT $(\mathrm{n}=3)$ & & & 0 \\
$\mathrm{CT}(\mathrm{n}=2)$ & $68 \pm 14$ & $36 \pm 13$ & transient phrenic \\
$\mathrm{SVC}(\mathrm{n}=1)$ & 29 & 13 & nerve palsies $(1)$ \\
\hline
\end{tabular}

AF: atrial fibrillation; Af: atrial flutter; AT: atrial tachycardia; $\mathrm{CCW}$ : counter clockwise isthmus-dependent; $\mathrm{CW}$ : clockwise isthmus-dependent; CT: crista terminalis; SVC: superior vena cava.
Table 3. Comparison of heart function before and after ablation.

\begin{tabular}{|c|c|c|c|c|}
\hline & -1 day & 7 days & 3 months & 6 months \\
\hline LVEDD (mm) & $51.7 \pm 4.5$ & $45.2 \pm 3.3^{\mathrm{a}}$ & $41.7 \pm 2.5^{\mathrm{abc}}$ & $40.5 \pm 3.1^{\mathrm{ab}}$ \\
\hline LVEF (\%) & $39.0 \pm 4.3$ & $45.6 \pm 3.5^{\mathrm{a}}$ & $51.5 \pm 2.7^{\mathrm{abc}}$ & $53.5 \pm 3.1^{\mathrm{ab}}$ \\
\hline 6-MWT (m) & $212 \pm 56$ & $262 \pm 47^{\mathrm{a}}$ & $305 \pm 37^{a b c}$ & $313 \pm 41^{\mathrm{ab}}$ \\
\hline $\mathrm{BNP}(\mathrm{ng} / \mathrm{L})$ & $3622 \pm 1860$ & $2429 \pm 1355^{\mathrm{a}}$ & $1530 \pm 866^{\mathrm{abc}}$ & $1300 \pm 520^{\mathrm{ab}}$ \\
\hline AHR (bpm) & $112.5 \pm 23.2$ & $73.3 \pm 15.3^{a}$ & $68.7 \pm 13.5^{\text {aec }}$ & $66.3 \pm 13.6^{\text {ae }}$ \\
\hline \multicolumn{5}{|l|}{ NYHA } \\
\hline IV+ III (n) & $17(68 \%)$ & $11(44 \%)^{\mathrm{a}}$ & $3(12 \%)^{\mathrm{abc}}$ & $2(8 \%)^{\mathrm{ab}}$ \\
\hline II (n) & $8(32 \%)$ & $14(56 \%)^{d}$ & $14(56 \%)^{\mathrm{aec}}$ & $13(52 \%)^{\mathrm{ae}}$ \\
\hline I (n) & $0(0 \%)$ & $0(0 \%)^{d}$ & $8(32 \%)^{\mathrm{abc}}$ & $10(40 \%)^{\mathrm{ab}}$ \\
\hline
\end{tabular}

${ }^{\mathrm{a}} P<0.05$ compared with -1 day; ${ }^{\mathrm{b}} P<0.05$ compared with 7 days; ${ }^{\mathrm{c}} P>0.05$ compared with 6 months; ${ }^{\mathrm{d}} P>0.05$ compared with -1 day; ${ }^{\mathrm{e}} P>0.05$ compared with 7 days; -1 day: one day before procedure; AHR: average heart rate.

symptom of heart failure [6]. Some studies proved that TIC was partially or completely reversible after normalization of the heart rate [7]. In patients with previous underlying IDC, persistent tachyarrhythmia plays a key role in the deterioration of cardiac function [8], therefore, in the management of refractory heart failure, much attention should be paid to heart rate. For patients without structural heart disease, prolonged tachyarrhythmia could result in development of left ventricular (LV) dysfunction [9], and a high suspicion of TIC is needed in the evaluation of a patient with new onset heart failure in the presence of atrial tachyarrhythmia. The diagnosis of TIC can be made when an improvement in function is seen after correction of arrhythmia [10]. The mechanism responsible for TIC has not been elucidated, and a number of theories have been proposed to explain the etiology of TIC, including depletion of high-energy phosphates, abnormal cellular remodeling and calcium handling $[11,12]$. It has also been proposed that chronic rapid heart rates could result in ischemia, which subsequently leads to reversible LV dysfunction due to myocardial shock or stunning [13].

In this study, 21 patients exhibited structural normal heart at first visit due to arrhythmia, and gradually developed symptoms of heart failure (HF) secondary to sustainted atrial tachyarrhythmia, only 4 patients previously suffered from IDC prior to occurrence of arrhythmia which consequently further aggravated HF. All subjects manifested remarkable improvement of LV function after resolution of atrial tachyarrhythmia, which supported the diagnosis of TIC because other forms of cardiomyopathy with poor LV function rarely demonstrated significant reverse of progression of disease within a short period of time [14]. It was reported that $\mathrm{AF}$ was the commonest cause of TIC, moreover, greater than $50 \%$ patients with $\mathrm{AF}$ and $\mathrm{LV}$ dysfunction who have some degree of TIC [15]. In our research AF accounted 
for $60 \%$ of all subjects with TIC.

In terms of treatment for $\mathrm{HF}$ patients with $\mathrm{AF}, \mathrm{AF}-$ FIRM study [16] demonstrated that pharmacological management for rhythm control was not superior to rate control in reducing mortality, hospitalization and NYHA functional class. However, rhythm control group was associated with increased hospitalization due to side effects of antiarrhythmic drugs. Catheter-based therapy for refractory $\mathrm{AF}$ in $\mathrm{HF}$ subjects has been an effective therapeutic option over the past decade, which exhibited better outcome than pharmacological therapy. Major procedures include atrio-ventricular node (AVN) ablation plus pacemaker implant and catheter-based rhythm control, radiofrequency ablation (RA) for pulmonary vein isolation (PVI) could restore atrioventricular and interventricular synchrony, in PABA study [17]. HF patients underwent PVI had better left ventricular function, 6 minutes walk distance and higher LVEF in contrast to AVN ablation with pacemaker implant at 6 months. RA has been proved to be the most efficient therapy to eliminate AF in HF patients so far, circumferential pulmonary vein isolation with additional line ablation has became the established approach [18] based on the finding that most cases of atrial fibrillation are triggered by ectopic foci originating from pulmonary veins [19]. Traditionally, for HF patients with AF, the therapeutic strategy is focused on correcting heart failure and lowering heart rate rather than converting AF, because it is commonly believed that left ventricular dysfunction plays a pivotal role in the initiation and maintance of AF [20]. This study demonstrated high success rate of RA in the treatment of AF in TIC patients including 4 subjects with underlying IDC, and RA could exert significant beneficial impact on left ventricular dysfunction recovery, which supported the fact that AF was an important component in the pathogenesis of TIC.

In one study [21] of patients with AF refractory to pharmacological treatment, catheter ablation was equally effective in patients with HF (LVEF $<45 \%$ with NYHA class II or higher symptoms) as it was in patients without HF, meanwhile, the LVEF was significantly increased by $21 \%$ at 12 months. Our study demonstrated $14.5 \%$ improvement of LVEF at the 6-month follow-up. Another clinical trial [22] indicated that ablation procedure was successful in maintaining sinus rhythm in $73 \%$ patients with impaired left ventricular function at 14 months, in our study, after completion of RA, only 4 patients suffered recurrence of arrhythmia which subsequently was controlled by amiodarone, the arrhythmia-free rate was $84 \%$.

Atrial flutter is another common atrial arrhythmia in clinical practice, compared with AF, which seems to be more susceptible to suffering from TIC due to more rapid ventricular response [23]. Our research revealed that all 7 atrial flutters were cavotricuspid isthmus-dependent. Typical isthmus linear ablation could successfully resolve atrial flutter. 3 subjects with TIC were identified as atrial tachycardia in EPS, in which 2 were CT origin and the other one arising from SVC, and these sites are common foci of atrial tachycardia [24]. In this study, only 2 patients receiving CPVI were detected mild pericardial effusion and $1 \mathrm{AT}$ case presented transient phrenic nerve palsies undergoing ablation within SVC, which proved $\mathrm{RA}$ was a relative safe procedure for TIC subjects.

In the follow-up, we observed that heart function improved dramatically within one week after the procedure. Before RA, a total of 17 subjects were classified as NYHA class IV and III, and 7 days later the number decreased to 11 , meanwhile, LVEDD reduced significantly from $51.7 \pm 4.5 \mathrm{~mm}$ to $45.2 \pm 3.3 \mathrm{~mm}$, which indicated rapid reverse remodeling of left ventricular after resolution of tachyarrhythmia. Other indexes referring to heart function including LVEF, 6-MWT and BNP simultaneously improved during this period. In the following 6 months, the above mentioned indexes further improved, however, there was no statistical significance between 3 and 6 months. Our results indicated that most patients could restore normal heart function within 3 months, even 4 subjects with underlying IDC benefited much from interruption of tachyarrhythmia, who demonstrated increased exercise capacity and resolution of heart failure symptoms after ablation. On admission, all of them were NYHA class IV, however, 7 days, 3 months and 6 months after the procedure, the numbers of NYHA class IV were 1,0 and 0 respectively. Our findings were compatible with the prior animal experiments, which proved that heart failure could result from rapid atrial pacing and prompt cessation of pacing could result in improvement of left ventricular function 1 day after termination and restore normal function within weeks [12].

In this study, all patients had received intensive medication for heart failure over 3 months on admission which was proved to be ineffective before ablation, one prominent characteristic of all subjects was poor-controlled ventricular response, and 24-hour ambulatory ECG monitor indicated average heart rate reached $112.5 \pm$ $23.2 \mathrm{bpm}$. After ablation, previous medical therapy remained unchanged in all patients, however, heart rate significantly decreased and was maintained in normal range with regular rhythm, within one week after procedure, reverse of heart failure could be observed in all affected patients, which proved that atrial arrhythmia with rapid and irregular ventricular response plays a central role in the pathogenesis and development of CIT.

Our study confirmed that CIT was a common form of left ventricular dysfunction in patients with atrial tachyarrhythmia, if pharmacological therapy could not resolve 
and slow the progression of heart failure, RA was an effective alternative.

\section{REFERENCES}

[1] Miyasaka, Y., Barnes, M.E., Gersh, B.J., Cha, S.S., Bailey, K.R., Abhayaratna, W.P., Seward, J.B. and Tsang, T.S. (2006) Secular trends in incidence of atrial fibrillation in Olmsted County, Minnesota, 1980 to 2000, and implications on the projections for future prevalence. Circulation, 114, 119-125. http://dx.doi.org/10.1161/CIRCULATIONAHA.105.5951 $\underline{40}$

[2] Bounhoure, J.P., Boveda, S., Albenque, J.P., et al. (2006) Arrhythmia-induced dilated cardiomyopathies. Bulletin del Academie de Nationale de Medecine, 190, 1225-1235.

[3] Ehrlich, J.R., Nattel, S. and Hohnloser, S.H. (2002) Atrial fibrillation and congestive heart failure: Specific considerations at the intersection of two common and important cardiac disease sets. Journal of Cardiovascular Electrophysiology, 13, 399-405. http://dx.doi.org/10.1046/j.1540-8167.2002.00399.x

[4] Grogan, M., Smith, H.C., Gersh, B.J. and Wood, D.L. (1992) Left ventricular dysfunction due to atrial fibrillation in patients initially believed to have idiopathic dilated cardiomyopathy. The American Journal of Cardiology, 69, 1570-1573. http://dx.doi.org/10.1016/0002-9149(92)90705-4

[5] De Ponti, R., Cappato, R., Curnis, A., et al. (2006) Transseptal catheterization in the electrophysiology laboratory: Data from a multicenter survey spanning 12 years. Journal of the American College of Cardiology, 47, 10371042. http://dx.doi.org/10.1016/j.jacc.2005.10.046

[6] Mclaran, C.J., Gersh, B.J., Sugrue, D.D., et al. (1985) Tachycardia induced myocardial dysfunction. A reversible phenomenon? British Heart Journal, 53, 323-327. http://dx.doi.org/10.1136/hrt.53.3.323

[7] Khasnis, A., Jongnarangsin, K., Abela, G., et al. (2005) Tachycardia-induced cardiomyopathy: A review of literature. Pacing and Clinical Electrophysiology, 28, 710721. http://dx.doi.org/10.1111/j.1540-8159.2005.00143.x

[8] Scheinman, M.M. (1998) Atrial fibrillation and congestive heart failure: The intersection of two common diseases. Circulation, 98, 941-942.

http://dx.doi.org/10.1161/01.CIR.98.10.941

[9] Umana, E., Solares, C.A. and Alpert, M.A. (2003) Tachycardia-induced cardiomyopathy. The American Journal of Medicine, 114, 51-55. http://dx.doi.org/10.1016/S0002-9343(02)01472-9

[10] Simantirakis, E.N., Koutalas, E.P. and Vardas, P.E. (2011) Arrhythmia-induced cardiomyopathies: The riddle of the chicken and the egg still unanswered?" Europace, 14, 466-473. http://dx.doi.org/10.1093/europace/eur348

[11] Fenelon, G., Wijns, W., Andries, E. and Brugada, P. (1996) Tachycardiomyopathy: Mechanisms and clinical implications. Pacing and Clinical Electrophysiology, 19, 95-106. http://dx.doi.org/10.1111/j.1540-8159.1996.tb04796.x
[12] Shinbane, J.S., Wood, M.A., Jensen, D.N., Ellenbogen, K.A., Fitzpatrick, A.P. and Scheinmann, M.M. (1997) Tachycardia-induced cardiomyopathy: A review of animal models and clinical studies. Journal of the American College of Cardiology, 29, 709-715. http://dx.doi.org/10.1016/S0735-1097(96)00592-X

[13] Moe, G.W., Montgomery, C., Howard, R.J., et al. (1993) Left ventricular myocardial blood flow, metabolism and effects of treatment with enalapril: Further insights into the mechanisms of canine experimental pacing-induced heart failure. Journal of Laboratory and Clinical Medicine, 121, 294-301.

[14] Iyer, V.R. (2008) Ventricular dysfunction: Tachycardiainduced cardiomyopathy. Indian Pacing and Electrophysiology Journal, 8, S122-S129.

[15] Redfield, M.M., Kay, G.N., Jenkins, L.S., et al. (2000) Tachycardia related cardiomyopathy: A common cause of ventricular dysfunction in patients with atrial fibrillation refered for AV node ablation. Mayo Clinic Proceedings, 75, 790-795. http://dx.doi.org/10.4065/75.8.790

[16] Freudenberger, R.S., Wilson, A.C. and Kostis, J.B. (2007) Comparison of rate versus rhythm control for atrial fibrillation in patients with left ventricular dysfunction (from the AFFIRM Study). American Journal of Cardiology, 100, 247-252.

http://dx.doi.org/10.1016/j.amjcard.2007.02.101

[17] Khan, M.N., Jais, P., Cummings, J., et al. (2008) Pulmonary-vein isolation for atrial fibrillation in patients with heart failure. The New England Journal of Medicine, 359, 1778-1785. http://dx.doi.org/10.1056/NEJMoa0708234

[18] Verma, A., Macle, L., Cox, J., et al. (2011) CCS Atrial Fibrillation Guidelines Committee. Canadian Cardiovascular Society atrial fibrillation guidelines 2010: Catheter ablation for atrial fibrillation/atrial flutter. Canadian Journal of Cardiology, 27, 60-66. http://dx.doi.org/10.1016/j.cjca.2010.11.011

[19] Bourke, J., Dunuwille, A., O’Donnell, D., et al. (2005) Pulmonary vein ablation for idiopathic atrial fibrillation: Six month outcome of first procedure in 100 consecutive patients. Heart, 91, 51-57. http://dx.doi.org/10.1136/hrt.2003.023093

[20] Ehrlich, J.R., Nattel, S. and Hohnloser, S.H. (2002) Atrial fibrillation and congestive heart failure: Specific considerations at the intersection of two common and important cardiac disease sets. Journal of Cardiovascular Electrophysiology, 13, 399-405. http://dx.doi.org/10.1046/j.1540-8167.2002.00399.x

[21] Hsu, L.F., Jais, P., Sanders, P., et al. (2004) Catheter ablation for atrial fibrillation in congestive heart failure. The New England Journal of Medicine, 351, 2373-2383. http://dx.doi.org/10.1056/NEJMoa041018

[22] Chen, M.S., Marrouche, N.F., Khaykin, Y., et al. (2004) Pulmonary vein isolation for the treatment of atrial fibrillation in patients with impaired systolic function. Journal of the American College of Cardiology, 43, 1004-1009. http://dx.doi.org/10.1016/i.jacc.2003.09.056

[23] Scheuemeyer, F.X., Grafstein, E., Heilbron, B., et al. (2011) Emergency department management and 1-year 
outcomes of patients with atrial flutter. Annals of Emergency Medicine, 57, 564-571.

http://dx.doi.org/10.1016/j.annemergmed.2010.09.027

[24] Zhao, Z., Li, X. and Guo, J. (2009) Electrophysiological characteristics of atrial tachycardia originating from the superior vena cava. Journal of Interventional Cardiac Electrophysiology, 24, 89-94.

http://dx.doi.org/10.1007/s10840-008-9324-0 